\title{
A RESPONSE TO FINKELSTEIN AND PIASETZKY'S CRITICISM AND “NEW PERSPECTIVE"
}

\author{
Amihai Mazar \\ The Institute of Archaeology, The Hebrew University of Jerusalem, Israel. Email: msmazar@mscc.huji.ac.il. \\ Christopher Bronk Ramsey \\ Research Laboratory for Archaeology and the History of Art, Oxford University, United Kingdom.
}

\begin{abstract}
The following short paper is a response to criticism by Finkelstein and Piasetzky (2010b), published in the present issue of Radiocarbon, of our 2008 paper in Radiocarbon concerning the evaluation of ${ }^{14} \mathrm{C}$ dates from Iron Age levels in Israel published by Boaretto et al. (2005). We refer to criticism concerning exclusion and inclusion of data. We also evaluate new models suggested by Finkelstein and Piasetzky and in particular their suggestion of regional stages marking the end of the Iron Age in Israel. We also comment on several methodological issues.
\end{abstract}

\section{INTRODUCTION}

One of the most important developments in historical archaeology of the southern Levant during the last decade was the wide-scale introduction of radiometric dates to the discussion. Frustration with the textual- (mainly biblical-) based interpretations of archaeological data led archaeologists to explore new avenues, and radiocarbon dating appeared to be a most helpful tool for objective dating, in particular in relation to the Iron Age, prior to $\sim 700$ BCE. In the Radiocarbon Conference proceedings published in 2001 (Radiocarbon, Volume 43), series of dates from Dor, Tel Rehov, and Beth Shean were the first relatively large groups of dates related to the Iron Age in Israel, measured thanks to collaboration in the 1990s between the ${ }^{14} \mathrm{C}$ laboratory at the Weizmann Institute of Science at Rehovot and the Israel Antiquities Authority. The Low Chronology for the Iron Age presented by Finkelstein (1996) spurred further research. The Iron Age Dating Project managed by Boaretto, Gilboa, Jull, and Sharon is a wide-scale project, where samples were collected from as many sites as possible, dated in 4 different laboratories, and emphasizing interlaboratory comparisons (Boaretto et al. 2005; Sharon et al. 2007). At Tel Rehov, over 60 samples were measured at the University of Groningen (Bruins et al. 2005) and at Khirbet en-Nahas a large-scale dating project has been ongoing in cooperation with Oxford University (Levy et al. 2008). The Yarnton conference titled "The Bible and Radiocarbon Dating" (Levy and Higham 2005) was another opportunity to publish large bodies of data and interpretations.

One of the main results of the first phase of the Iron Age Dating Project (Sharon et al. 2007) study, achieved with the use of Bayesian models prepared with OxCal software (Bronk Ramsey 1995, 2001) was that the transition from Iron I to Iron II should be lowered from 1000 BCE (traditional dating) to $\sim 900 \mathrm{BCE}$, in agreement with Finkelstein's Low Chronology. This result could be of utmost importance for historical interpretation, since it would mean that archaeological assemblages attributed traditionally to the 10th century (the assumed time of the United Monarchy of David and Solomon according to the inner-biblical chronology) date instead to the 9th century, and have nothing to do with the United Monarchy. In our previous paper (Mazar and Bronk Ramsey 2008; see also Mazar 2008 with excursus written in cooperation with Bronk Ramsey), we attempted to show that:

1. Bayesian models using the same data with several different evaluations of outliers indicate a date for the transition from Iron I to Iron II as follows: 962-942 BCE (68.2\%) or 966-929 BCE (95/4\%) including wood samples; 948-919 BCE (68.2\% or 963-913 [95.4\%]) using only shortlived material (Mazar and Bronk Ramsey 2008:175).

2. The transition between the 2 periods was probably a long process that lasted several decades. 
3. Heavy destructions which mark the end of several major Iron Age I sites in Israel occurred during a time range covering the last decades of the 11th century and first decades of the 10th century BCE, no later than $960 \mathrm{BCE}$. Thus, the end of the Iron Age I and transition to Iron Age II must have started during those years.

4. Outliers exist in all measurements and should be evaluated in a proper manner because a small number of aberrant results can change the whole picture when the calendar precision required is as great as it is here.

Several points in our 2008 paper were criticized by Finkelstein and Piasetzky (2010b), who also suggest a new way to understand the transition from Iron Age I to Iron Age II in Israel. In this new suggestion, some of the basic ideas of the Low Chronology are dropped (Finkelstein and Piasetzky in this issue and 2009; and see below). We are grateful to Editor Tim Jull of Radiocarbon for the opportunity to respond to their paper.

Our response is divided into 2 parts:

1. Response to the critique of data inclusion or exclusion in our paper.

2. Evaluation of Finkelstein and Piasetzky's new suggestion concerning the transition from Iron I to Iron II. We think there is insufficient evidence for their suggested stages in this transition. However, we think that their overall conclusion is similar to ours and that this might have important consequences for historical interpretation of the archaeology of the region.

We also add a few methodological comments concerning the use of radiometric dates. The following discussion is structured following Finkelstein and Piasetzky's (2010b) paper in the current issue and cites the page numbers therein.

\section{COMMENTS ON “METHODS, DATA INCLUSION, AND EXCLUSION"}

\section{“Methods" (p 1667)}

We agree with much of what was written in this section, but we do not feel that it should have been directed at our paper because, as we explained therein, it was not an attempt at a comprehensive study of the corpus of radiometric dates from the Iron Age, but rather an evaluation of the data published in Boaretto et al. (2005). We therefore referred to all the data in that article, with some updates based on Sharon et al. (2007), which was published when our paper was in the editing process. ${ }^{1}$ Consequently, the data selected for our paper was dictated by the paper to which we responded, with the exception of a single date from Tell Qasile. As for the issue of wood samples also raised by Finkelstein and Piasetzky in this section, see below. We have, however, reservations concerning the authors' statement that all ${ }^{14} \mathrm{C}$ determinations from reliable contexts should be included in statistical studies. In such a subtle chronological debate, with less than a century dividing the various views,

\footnotetext{
${ }^{1}$ Sharon et al. (2007) appeared when Mazar and Bronk Ramsey (2008) had already been submitted and our Bayesian model already constructed based on data in Boaretto et al. (2005). While updating our paper, we cited only those samples from Sharon et al. (2007) relevant to the transition Iron I/Iron II, which was the focal issue of our paper. Samples not used by us would not change the results. These include: Late Bronze period: samples 4274 and 4510 from Tel Zayit and Aphek); Iron IA: Sample 4501 from Megiddo K-6; Iron I: samples 4270-4273 from el Ahawat (pottery assemblage not yet published); sample 4147 from Tel Hebron (omitted by mistake - a second identical sample 4148 was included); samples 3934-3936 from Beth Shemesh Strata 5 and 6 (early and middle Iron I); samples 4522, 4525, 4528 from Tel Dor D2/12 (middle Iron I); samples 4283 and 4284 from Tel Miqne VI-V (middle Iron I). Iron IIA: Samples 3989-3991 from Sulem (context not published); samples 4583-4587 from Moza (context not published); Iron IIB: Samples 3931, 3937, 3938 from Beth Shemesh 3 (this level continues from Iron IIA to Iron IIB according to the excavators); 4275, 4278, 4279, 4280: Tel Zayit; 4422 Tell el Hamma. Insecure contexts: sample 4288 from Tel Miqne; sample 3806 from Tel Rehov.
} 
we recommend that only the highest quality measurements available be used, together with some method for excluding outliers that might arise due to inappropriate sample materials, problems with contextual interpretations, or measurement problems.

\section{"Unjustified Exclusion of Data" (p 1668)}

Tel Miqne-Ekron and Beth Shemesh. The 2 samples 4283 and 4284 from Tel Miqne Strata VI and V and the 3 samples from Beth Shemesh Strata 6 and 5 were mostly treated on p 161, Note 2 in Mazar and Bronk Ramsey (2008); they had not been included in Boaretto et al. (2005) but were published in Sharon et al. (2007), to which we referred only partly. Perhaps these samples should have been added to our data, although they would not have changed the results, since they fit the conventional chronology for the Iron Age I.

Rehov and Dor. In light of the above-mentioned aim of our paper, we only used dates from Tel Rehov and Dor that were included in Boaretto et al. (2005). The many other dates from both these sites measured in the framework of earlier projects were published and discussed in detail elsewhere and so deliberately were not included in our data (the references are cited in our 2008 paper).

Tel Hadar. We deliberately used only those dates from Tel Hadar that were measured in the framework of the recent Iron Age Dating Project and published by Boaretto et al. (2005) and Sharon et al. (2007). Many of the dates used by Finkelstein and Piasetzky (2010b) in their response were published for the first time on this occasion (except for a general statement that provided their average; see Mazar and Bronk Ramsey 2008:161, Note 2, where we commented on this subject). These 12 dates, listed as HD101, do not form a consistent group. If one assumes that they are all contemporary and tries to combine them, they fail a chi-squared test $(d f=11, t=33.512$ : compared to 19.7 for $p=$ $5 \%$ ) This must either be because they do not all relate to the same event or because some of the measurements are significant outliers.

El-Ahwat: Sharon et al. (2007). Table 8 lists 2 dates from the site that are mainly in the 11th century and 2 others that are mainly in the 10th century. An evaluation of these data will be possible only after the archaeological assemblage is published, as explained in our 2008 paper, p 161, Note 2.

Tell el-Hammah early phase. Samples 4416 and 4417 from Hammah do appear in our previous paper (p 165, Table 2). It should be noted that Finkelstein and Piasetzky omitted sample 4416 from the same context in their database. On p 167 of our article, we explained that the 12 pottery sherds published from this level could belong either to the late Iron I or the early Iron IIA, and thus the attribution of this level to one of these periods remains questionable. As for the charge that we "exclude the readings for the upper Iron IIA destruction" at Tel el-Hammah, Finkelstein and Piasetzky are probably referring to sample 4422 from Locus 205 defined in Sharon et al. (2007:39) as "IronIIA/ B." Locus 205 is not mentioned in the currently available publication on Tell el- Hammah, no pottery from this context has been published, and the date $(2588 \pm 18)$ points to the Iron IIB (8th century $\mathrm{BCE})$, which was beyond the scope of our paper.

Hazor. On page 171 of our Mazar and Bronk Ramsey (2008), we explained at length why samples 3784 and 3786 included in Sharon et al. (2007) must be outliers.

\section{“Problematic Inclusion of Data" (p 1668-1670)}

Charcoal. Finkelstein and Piasetzky dedicate a lengthy but unnecessary discussion to the "old wood" effect. We are of course aware of this effect, and therefore presented alternative models, one with and one without charcoal (see Mazar and Bronk Ramsey 2008:173, models B3 and C3; a sim- 
ilar practice was used in Sharon et al. 2007). This was well known to the authors, since they cite our alternative models, and their criticism is therefore unwarranted. This said, we still justify the inclusion of the charcoal dates in our data for of 2 reasons: (1) as mentioned above, our article was a response to Boaretto et al. (2005), which did include charcoal; and (2) regarding the specific case of Hazor XII/XI, on p 163, we emphasized the gap between the conventional archaeological dates (12th-11th centuries BCE) and the ${ }^{14} \mathrm{C}$ dates of both the charcoal and the 2 short-lived samples from this level. Explanations for this phenomenon may be either that all samples from the Stratum XII/XI pits originated in the Late Bronze Age stratum into which the pits were cut, or that Stratum XII/XI is earlier than our conventional archaeological dating.

Hebron and Bethsaida were cited in our paper despite the lack of publication of the pottery due to A Mazar's personal familiarity with these assemblages, which allowed for an evaluation of their attribution to the Iron I and Iron IIA, respectively.

\section{COMMENTS ON “ALTERNATIVE BAYESIAN MODELS: MODEL I” (p 1670-1676)}

Although Finkelstein and Piasetzky's (2010b) basic approach does not differ substantially from our own models, we wish to comment on several of the selected sites used in their models.

End of the Iron I (Table 2 in Mazar and Bronk Ramsey 2008). For the early phase at Tell el-Hammah and for Tel Hadar, see our comments above. Tel Rehov local Stratum D-3 is represented by a layer of pits uncovered in a limited area, which cut into substantial buildings of local Stratum D-4, the last Iron Age I city. In other parts of the site in which the last Iron Age I city was detected (in Areas B and C), no such pits were found, and it seems that Stratum D-3 is a local phenomenon limited to only 2 excavation squares in Area $\mathrm{D}$ and representing a short-lived activity between the end of the last Iron I city and the establishment of Stratum VI (site-wide stratum number) of the early Iron IIA. Since several ${ }^{14} \mathrm{C}$ determinations clearly date local Stratum D-4 to the 11 th century BCE (Mazar et al. 2005:199), it seems that the Stratum D-3 pits should be dated to the earlier part of their radiometric range, namely, $\sim 1000$ BCE. In their list of dates from Stratum D-3 at Tel Rehov, Finkelstein and Piasetzky include sample RW 3120a (published by Mazar and Carmi 2001:1336), which is much later than the other dates from this stratum and should be regarded as an outlier, as mentioned already in the original publication. In general, we do not think that a policy of including all dates that are within $5 \sigma$ of the mean is justifiable - samples should only be more than $5 \sigma$ away from the mean in less than 1 in a million cases (contra Finkelstein and Piasetzky 2009:258). Such outliers might seriously bias the models. A better approach for the future might be to use outlier analysis (Bronk Ramsey 2009), since this allows for the down-weighting of outliers without having to identify them individually.

Early Iron IIA (Table 3 in Mazar and Bronk Ramsey 2008). Finkelstein and Piasetzky's attempt to isolate "Early Iron IIA" contexts may be justified, but such contexts are extremely rare. Of the 5 site contexts they define as belonging to this horizon, we would exclude at least two: Lachish V and Aphek X-8, the attribution of which to either the early or late Iron IIA remains unclear. It should be emphasized that a good number of other Iron IIA contexts yielded dates that are similar or even earlier than those mentioned by the authors, for example, 1 of the 2 dates of short-lived samples from Megiddo VA-IVB (No. MG13-16 Mazar and Bronk Ramsey 2008:168, Table 3). In our work, we included all the Iron Age IIA contexts in 1 group, which indeed may create a problem for the Bayesian model, since there is no question in our view that this period lasted over $150 \mathrm{yr}$, and a bias may be created by the later contexts within the period. However, deciding what to include and what to exclude from a presumed "Early Iron IIA" context is not a simple task. 


\section{COMMENTS ON “MODEL II” (p 1676-1679)}

Finkelstein and Piasetzky (2010b) followed our method for determining the end of the Iron Age I by means of the dates of major destruction events that are attributed to the end of this period. They agree with us that the major destructions - of Megiddo VIA, Yoqne'am XVII, and Tell Keisan 9occurred in the early 10th century BCE, according to our calculations, between the last decades of the 11th and the early to mid-10th century (see Mazar and Bronk Ramsey 2008:176, 178, Figure 4). Finkelstein and Piasetzky present a more precise date of $2852 \pm 13 \mathrm{BP}$, which must be the combination of many dates, all assumed to be from the same event. This date calibrates to 1086-934 cal BC at $95 \%$ probability (or $1047-996$ cal $\mathrm{BC}$ at $68 \%$ probability), which is not significantly different than our results. We would argue that both our and Finkelstein and Piasetzky's analyses suggest that the underlying changes in the material culture between the Iron I and the Iron IIA take place early in the 10th century. Of course, the transition from the Iron I to the Iron II did not necessarily occur simultaneously across the region and it could have been a much more complex process. The issue seems to be almost semantic: where does one put the "transition" label in a process that started in the early 10th century and was largely completed in the latter half of the same century?

Finkelstein and Piasetzky's (2010b) suggestion that 3 regional "ends of Iron I" be distinguished needs to be evaluated critically (see their Figures 6-7 and Table 5; see also Finkelstein and Piasetzky 2009:267-70). First, they attempt to distinguish between "western" and "eastern" destructions in the northern valleys of Israel. Of the 3 sites used as type-sites for the "eastern destructions," however, the only one that can be taken into account is Tel Hadar (see above for the early phase at Tell elHammah and for Tel Rehov D-3; the latter two have no destruction layer anyway). Using only the high-quality dates from Tel Hadar V published in Sharon et al. (2007), we calculated the time range for its destruction at 1043-979 BCE (see Mazar and Bronk Ramsey 2008:167, 177, Figure 3D); this is no later than the destructions of the "western" sites, like Megiddo VIA. Thus, the thesis of a "gradual demise of the Iron I culture in the northern valleys" is highly questionable. The same applies to the third region, the "coastal" region, which is based on 2 sites: Tel Dor D2-10-9 and Tell Qasile X. No destruction level from this period is reported at Dor. The great destruction at Tell Qasile recalls that of Megiddo VIA in both nature and dating: 1039-979 BCE (68\% probability; see Mazar and Bronk Ramsey 2008:167, 177, Figure 3C). Thus, Finkelstein and Piasetzky's suggested pattern of regional differences in the dates of the destructions at the end of the Iron Age I is not supported by the evidence. According to our calculations, the lowest possible date for all these destructions at the end of the Iron I is about $960 \mathrm{BCE}$; higher dates closer to $\sim 1000 \mathrm{BCE}$ are plausible, since the possible time range for these destructions starts comfortably in the 11th century BCE.

In any event, an important point of agreement between us is the destruction date of Megiddo VIA and adjacent sites during the first decades of the 10th century BCE. This is in contrast to Finkelstein's earlier views: in his basic paper representing his Low Chronology he claimed that Megiddo VIA was destroyed by Shoshenq (Finkelstein 1996:183). In 2002, this claim was moderated: he now claimed that Shoshenq may have destroyed Megiddo VIA, but he leaves an option that Megiddo VIA was destroyed earlier in the 10th century BCE (Finkelstein 2002:117-22) and in 2006 and later he and Piasetzky prefer the earlier date in the 10th century, based on ${ }^{14} \mathrm{C}$ dates (Finkelstein and Piasetzky 2006:58), a date with which we agree. The destruction of Megiddo VIA is fundamental to our subject, since it correlates with other violent destructions (like Yokneam XVII, Tell Keisan 9) that mark the end of the Iron Age I. Tell Qasile X and Tel Hadar V provide additional support for this date with their own ${ }^{14} \mathrm{C}$ dates mentioned above.

Now that all four of us agree on this subject, it becomes clear that the transition from the Iron I to Iron II must have started in the first half of the 10th century BCE, in clear contrast to a fundamental 
claim of Finkelstein's Low Chronology, which dates this transition to around 900 BC (e.g. Finkelstein 1996:182). This is now also confirmed at the fortified early Iron IIA site of Khirbet Qeiyafa in the Judean Shephelah, where $4{ }^{14} \mathrm{C}$ dates of short-lived samples measured at Oxford point to the date $2844 \pm 15$ (1051-969 BCE at 77.8\% probability) (Garfinkel and Ganor 2009:35-8). This date more or less corresponds to the dates of the above-mentioned destructions in the northern valleys and along the coast. All of these radiometric dates provide a time range of some $\pm 25 \mathrm{yr}$. We agree with Finkelstein and Piasetzky (2010b) that the transition from the Iron I to Iron II could have been a rather lengthy process with different regional developments, but the best available dates for the beginning of these developments are the wave of destructions that mark the end of Iron Age I and the initial beginning of the new Iron IIA material culture, as detected at sites like Khirbet Qeiyafa, Tel Rehov (Stratum VI), and others. We should also mention the large number of dates from the copper extraction site of Khirbet en-Nahas, dating a local version of Iron IIA material culture to the 10th-9th centuries BCE (Levy et al. 2008).

It remains to be asked why Bayesian models may arrive at dates that differ by up to $40-50 \mathrm{yr}$. In our view, there are 2 reasons: (1) the relative lack of dates from the early part of Iron IIA; and (2) the choices of sites and dates selected for the construction of the models. In order to resolve these issues, we must await additional data, such as those expected from the second phase of the Iron Age Dating Project directed by Boaretto, Gilboa, and Sharon, as well as from other sites. It is also important that biases in the data are properly treated in the models (as we attempted to do by treating the late Iron I material separately), and that extreme outliers are excluded, since these can have a disproportionately large impact on the model outputs.

The main message of our 2008 paper was that the data presented in Boaretto et al. (2005) and Sharon et al. (2007) (the latter used only partly in our study) could not be used as incontrovertible evidence for the Iron I/II transition having taken place at the end of the 10th century BCE. In their response to our paper, Finkelstein and Piasetzky (2010b) indeed seem to accept that in some regions, the end of the Iron I occurred significantly earlier in the 10th century, and we suggest that this conclusion can be extended over larger parts of the country, in line with the conventional chronology. We still do not know how rapidly this process was completed and what specific regional pattern, if any, can be established.

\section{REFERENCES}

Boaretto E. 2006. Radiocarbon dates. In: Finkelstein I, Ussishkin D, Halpern B, editors. Megiddo IV: The 1998-2002 Seasons. Tel Aviv: Tel Aviv University. p 550-7.

Boaretto E, Jull AJT, Gilboa A, Sharon I. 2005. Dating the Iron I/II transition in Israel: first intercomparison results. Radiocarbon 47(1):39-55.

Bronk Ramsey C. 1995. Radiocarbon calibration and analysis of stratigraphy: the OxCal program. Radiocarbon 37(2):425-30.

Bronk Ramsey C. 2001. Development of the radiocarbon calibration program. Radiocarbon 43(2A):355-63.

Bronk Ramsey C. 2009. Dealing with outliers and offsets in radiocarbon dating. Radiocarbon 51(3):1023-45.

Bruins HJ, van der Plicht J, Mazar A, Bronk Ramsey C, Manning SW. 2005. The Groningen radiocarbon series from Tel Rehov: OxCal Bayesian computations for the Iron IB-IIA boundary and Iron IIA destruction events. In: Levy TE, Higham T, editors. The Bible and
Radiocarbon Dating: Archaeology, Text and Science. London: Equinox. p 271-93.

Finkelstein I. 1996. The archaeology of the United Monarchy: an alternative view. Levant 28:177-88.

Finkelstein I. 2002. The campaign of Shoshenq I to Palestine: a guide to the 10th century BCE polity. Zeitschrift des Deutschen Palästina-Vereins 118:10935.

Finkelstein I, Piasetzky E. 2006. The Iron I-IIA in the highlands and beyond: ${ }^{14} \mathrm{C}$ anchors, pottery phases and the Shoshenq I campaign. Levant 38:45-61.

Finkelstein I, Piasetzky E. 2009. Radiocarbon-dated destruction layers: a skeleton for Iron Age chronology in the Levant. Oxford Journal of Archaeology 28(3): 255-74.

Finkelstein I, Piasetzky E. 2010a. Radiocarbon dating the Iron Age in the Levant: a Bayesian model for six ceramic phases and six transitions. Antiquity 84(324): 374-5. 
Finkelstein I, Piasetzky E. 2010b. The Iron I/IIA transition in the Levant: a reply to Mazar and Bronk Ramsey and a new perspective. Radiocarbon 52(4):1667-80.

Garfinkel Y, Ganor S. 2009. Khirbet Qeiyafa Volume I: Excavation Report 2007-2008. Jerusalem: Israel Exploration Society.

Levy T, Higham T, editors. 2005. The Bible and Radiocarbon Dating: Archaeology, Text and Science. London: Equinox.

Levy TE, Higham T, Bronk-Ramsey C, Smith NG, Ben Yosef E, Robinson M, Munger S, Knabb K, Schultz Najjar M, Tauxe L. 2008. High-precision radiocarbon dating and historical biblical archaeology in southern Jordan. Proceedings of the National Academy of Science 105(43):16,460-5.

Mazar A. 2008. From 1200 to 850 B.C.E.: remarks on some selected archaeological issues. In: Grabbe L, editor. Israel in Transition: From Late Bronze II to Iron
IIa (c. $1250-850$ B.C.E.). New York: T\&T Clark. p 86-121.

Mazar A, Bronk Ramsey C. 2008. ${ }^{14} \mathrm{C}$ dates and the Iron Age chronology of Israel: a response. Radiocarbon 50(2):159-80.

Mazar A, Carmi I. 2001. Radiocarbon dates from Iron Age strata at Tel Beth Shean and Tel Rehov. Radiocarbon 43(3): 1333-42.

Mazar A, Bruins HJ, Panitz-Cohen N, van der Plicht J. 2005. Ladder of time at Tel Rehov: stratigraphy, archaeological context, pottery and radiocarbon dates. In: Levy TE, Higham T, editors. The Bible and Radiocarbon Dating: Archaeology, Text and Science. London: Equinox. p 193-255.

Sharon I, Gilboa A, Jull AJT, Boaretto E. 2007. Report on the first stage of the Iron Age Dating Project in Israel: supporting a Low Chronology. Radiocarbon 49(1):146.

\section{ADDENDUM}

by Amihai Mazar

The models represented by Finkelstein and Piasetzky in their response in this issue (Finkelstein and Piasetzky 2010b) are not supported by detailed data. But in a recent paper in Antiquity (Finkelstein and Piasetzky 2010a), similar models are represented with detailed supporting data provided online. In their Data Table 6 (Finkelstein and Piasetzky 2010a), it is clear that they mistakenly include data from Tel Rehov Stratum V by mixing secure loci from this stratum with loci defined by the excavators as belonging to either Stratum V or Stratum IV.

In Table 1, we compare dates of 5 samples from secure loci of Stratum $\mathrm{V}$ in Area $\mathrm{C}$ at Tel Rehov (where this stratum was best defined and isolated). The first 3 samples come from the destruction layer of the apiary discovered in this level. From each sample, 3-6 duplicates were measured and the averages are cited here as published. The dates all overlap the 10th/9th centuries BCE; some are more likely to lie in the 10th century and some in the 9th century. The pottery assemblage from this level is identical to that of Stratum IVB-VA at Megiddo, which was identified as "Solomonic" (10th century) by Y Yadin and as "Omride" (9th century) by Finkelstein. The 2 available samples from Tel Aviv University Megiddo H-5, correlated by the excavators with Stratum IVB-VA of the Chicago expedition, are cited in Table 1. Sample 3949 (with 4 repetitions) is clearly dated to the 10th century BCE, while the single measurement of sample 3948 is more likely to lie in the 9th century BCE (Boaretto 2006:555 and Table 1 below).

The results from these 2 sites indicate a possibility that some of these contexts (defined as "Late Iron IIA") date from the second half of the 10th century BCE. 


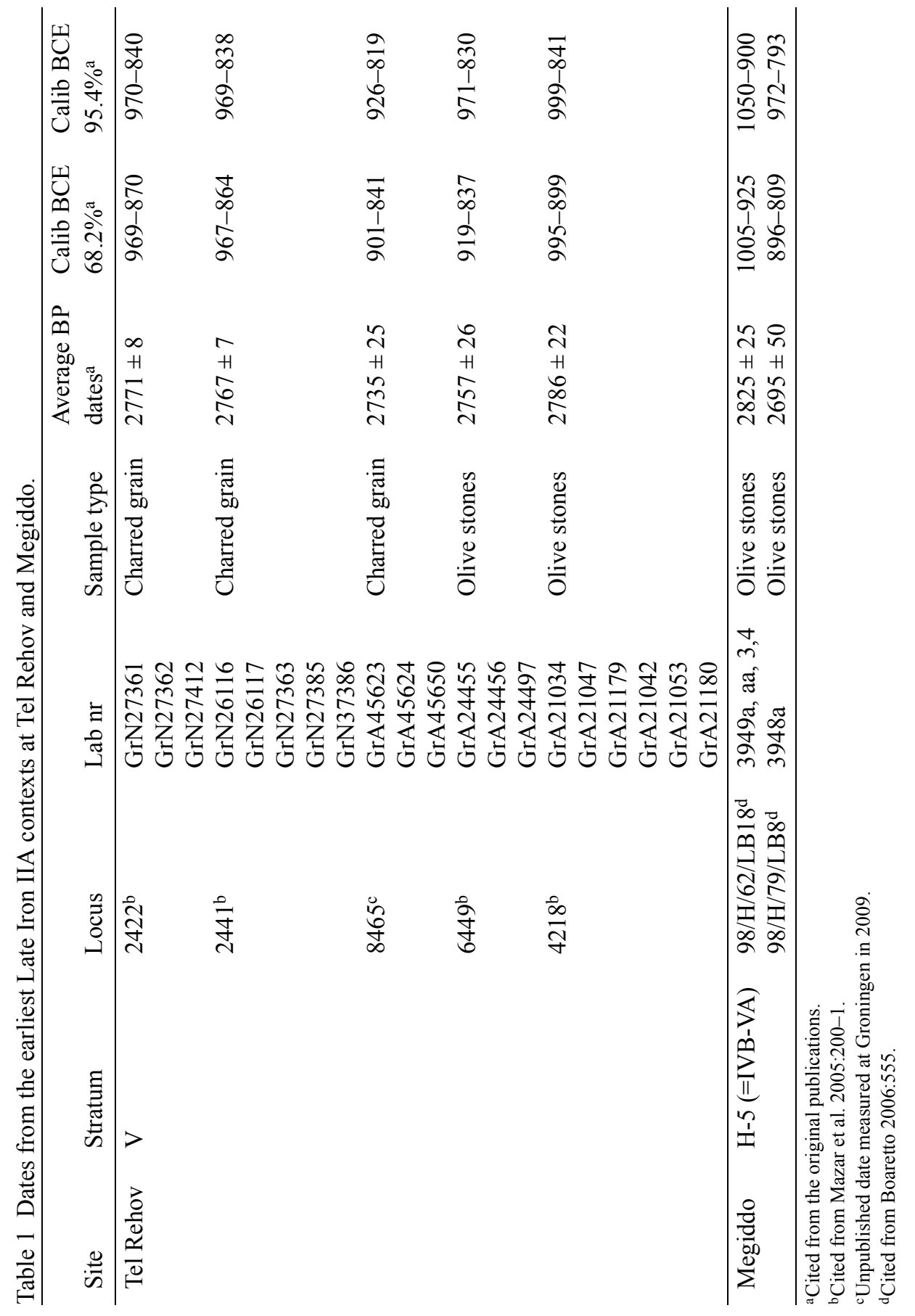

